Check for updates

Cite this: RSC Adv., 2019, 9, 5076

\title{
A contact lens promising for non-invasive continuous intraocular pressure monitoring
}

\author{
Yu Pang, (D) ab Yuxing Li, ${ }^{\text {ab }}$ Xuefeng Wang, ${ }^{\text {ab }}$ Chenjie Qi, ${ }^{c}$ Yi Yang ${ }^{\text {ab }}$ \\ and Tian-Ling Ren (iD *ab
}

Intraocular pressure (IOP) is the key indicator to assess the risk for and status of glaucoma patients, and medication at high IOP values slows down exacerbation of glaucoma. However, the IOP value is subject to circadian variation and cannot be measured continuously in clinical practice. Herein, we have fabricated a contact lens with a Wheatstone bridge circuit for non-invasive monitoring of IOP. A metal electrode has been used as a strain gauge to measure the weak deformation of the eyeball caused by IOP variation. Tests on a model eye indicate that the contact lens exhibits a high sensitivity of $20 \mu \mathrm{V}$ $\mathrm{mmHg}^{-1}$ and excellent dynamic cycling performance at different speeds of IOP variation. These results demonstrate that the contact lens sensor is promising for continuous IOP monitoring of glaucoma disease, regardless of the posture and activities of the patient.

Received 14th December 2018 Accepted 23rd January 2019

DOI: $10.1039 / \mathrm{c} 8 \mathrm{ra10257k}$

rsc.li/rsc-advances
IOP monitoring owing to the requirements for precise measurements and expert operation. Therefore, it is urgent to develop simple, low-cost and continuous IOP monitoring techniques, especially wearable sensors. So far, several methods based on the working principles of hydrodynamics, ${ }^{9}$ capacitive and inductive reactance, ${ }^{10-13}$ and piezo-resistive effect ${ }^{14-17}$ have been investigated to meet those new requirements. Araci et al. have developed an implantable contact lens with an airtight microfluidic channel, but it is difficult to read the pressure through a hazy cornea. ${ }^{9}$ Via a change in the distance between capacitive electrodes or the length in inductance coils, the IOP pressure can be detected and received by an external oscillator circuit. Capacitive IOP sensors with a sensitivity of $160 \mathrm{kHz}$ $\mathrm{mmHg}^{-1}$ and a sensitivity of $15 \mathrm{kHz} \mathrm{mmHg}^{-1}$ were reported in 2009 and 2013, respectively. ${ }^{\mathbf{1 0 1 1}}$ However, both sensors need surgery to implant them inside the eyes. In addition, piezoresistive sensors provide promising non-invasive techniques to detect the curvature variation of the cornea. By using a conducting polymer bi-layer film, Laukhin et al. have fabricated a strain sensor over the whole area of a contact lens with good linearity and reproducibility but a low sensitivity of $1.5 \Omega$ $\mathrm{mmHg}^{-1} \cdot{ }^{\mathbf{1 4}}$ Renaud and co-workers have fabricated an IOP contact lens with a sensitivity of $8.37 \mu \mathrm{V} \mathrm{mmHg}{ }^{-1} \cdot{ }^{16}$ However, the use of nontransparent materials (polycarbonate and polyimide) in these contact lenses would greatly influence the visual field during long-term IOP monitoring. Moreover, the sensitivity and dynamic response of the output in response to the IOP variation need to be improved for potential practical application.

In this work our effort was to develop a non-invasive contact lens with high sensitivity, transmittance and linearity for 24

hour IOP monitoring. A Wheatstone circuit was designed to

\footnotetext{
${ }^{a}$ Institute of Microelectronics, Tsinghua University, 100084, Beijing, China. E-mail: rentl@tsinghua.edu.cn

${ }^{b}$ Beijing National Research Center for Information Science and Technology (BNRist), Tsinghua University, 100084, Beijing, China

'School of Applied Science, Beijing Information Science \& Technology University, 100192, Beijing, China
} 
improve the precision and eliminate the temperature drift. Using transparent polyethylene terephthalate (PET) as substrate, two counterpart active strain gauges and two counterpart passive strain gauges were embedded in the contact lens. The sensor shows a sensitivity of $20 \mu \mathrm{VmmHg}{ }^{-1}$ within the large range of $9-30 \mathrm{mmHg}$ on a polymer eyeball model. Besides this, it exhibits outstanding response to different speeds of IOP change and a high linearity of 0.996 . The contact lens sensor could allow continuous IOP monitoring regardless of the position and activities of a patient, and displays promise for point-of-care management in glaucoma patients.

\section{Design and fabrication}

Generally, an IOP change of $1 \mathrm{mmHg}$ would cause a change of central corneal curvature of about $3 \mu \mathrm{m}$ (for a corneal curvature of $7.8 \mathrm{~mm}),{ }^{18}$ which can induce a small expansion or constriction of the corneal perimeter. A piezo-resistive strain gauge was designed to detect the weak deformation which leads to a resistance increase or decrease. A Wheatstone bridge is adopted to measure the weak signal variation, having the advantage of high sensitivity and precision; see Fig. 1a. The bridge contains a counter pair of measuring resistances $\left(R_{2}, R_{3}\right)$ and a counter pair of fixed resistances $\left(R_{1}, R_{4}\right)$. If a constant voltage is supplied to the bridge, the output voltage $V_{\mathrm{t}}$ can be expressed by the following equation: ${ }^{19}$

$$
V_{\mathrm{t}}=\frac{R_{2} R_{4}-R_{1} R_{3}}{\left(R_{1}+R_{2}\right) \times\left(R_{3}+R_{4}\right)} V_{0}
$$

where $V_{0}$ is the supplied voltage, and $R_{1}, R_{2}, R_{3}$ and $R_{4}$ are four loading resistances. To simplify the expression, the four initial resistances can be designed to be the same value $\left(R_{1}=R_{2}=R_{3}=\right.$ $R_{4}=R$ ), namely by using the same electrode length for each. Moreover, to eliminate the effect of initial $R$ on the output voltage and get a linear relationship between output voltage and resistance variation, a constant current is supplied to the Wheatstone bridge. As shown in Fig. $1 \mathrm{~b}$, for IOP variation the output voltage caused by the active gauge can be expressed by the following equation:

$$
\frac{V_{\mathrm{t}}}{I_{0}}=\frac{\Delta R}{2}=\rho \frac{\pi \Delta d}{2 W L}
$$

where $\Delta R$ is the variation in resistance, $I_{0}$ is the constant current supply, $\rho$ is the specific resistance, $\Delta d$ is the change in diameter, and $W$ and $L$ are the width and length of the cross-section coil which are considered as constant values under weak deformation.

The change in diameter, $\Delta d$, is determined by the pressure variation in the eyes, which relates to the physical parameter of the eyeball. ${ }^{13}$ Note that the arc length of the contact lens remains the same for the pressure increasing and decreasing. Thus, $\Delta d$ can be expressed by the equation:

$$
\frac{\Delta d}{2}=(r+\Delta r) \frac{\sin \alpha r}{2(r+\Delta r)}-r \frac{\sin \alpha}{2}
$$

where $r$ is the initial radius of the contact lens, $\Delta r$ is the radius change caused by the IOP, and $\alpha$ is the initial opening angle of the measuring gauge. Considering that $\Delta r$ is far less than $r$
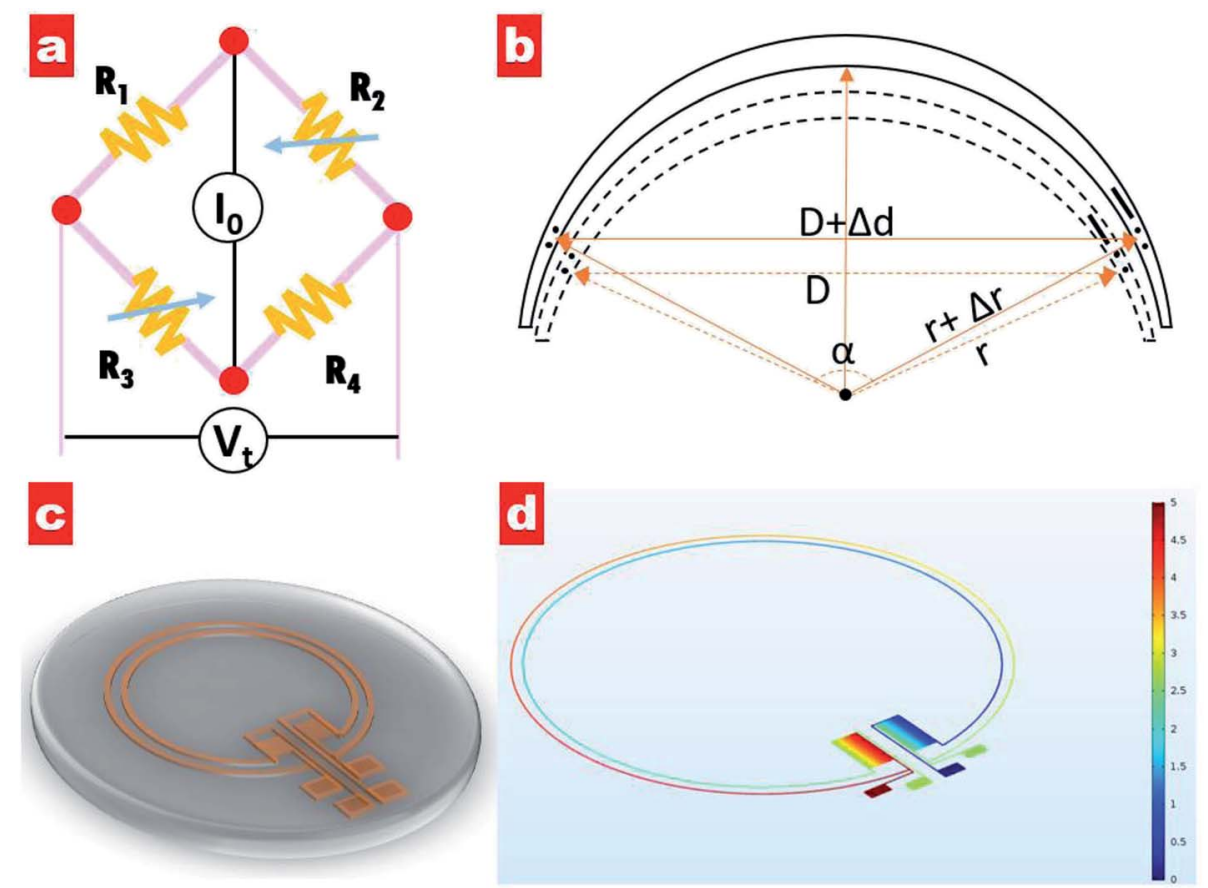

Fig. 1 (a) The Wheatstone bridge circuit for the eyeball pressure sensor, which contains one counter pair of measured resistances and one counter pair of fixed resistances. (b) A schematic diagram of the deformation of the contact lens induced by IOP change, which leads to curvature radius changes $\Delta r$. (c) A schematic diagram of the contact lens sensor, which contains two active strain gauges and two passive resistances. (d) The simulation result of potential distribution with a constant voltage supply; the color bar key represents the voltage distribution from 0 to $5 \mathrm{~V}$. 
Table 1 Piezo-resistive contact lens design parameters

Diameter of the inside strain gauge

Diameter of the outside strain gauge

Width of the strain gauge

Diameter of the contact lens

Radius of curvature of the contact lens

Central thickness of the contact lens

under weak deformation, the above equation can be simplified as:

$$
\Delta d \approx A \Delta r
$$

where $A$ is a constant, equal to $2 \sin (\alpha / 2)-\alpha \cos (\alpha / 2)$. Moreover, the IOP change generates a certain variation of corneal curvature, and thus $\Delta r$ can be expressed by the following equation:

$$
\Delta r=B \Delta p
$$

where $B$ is a constant which is dependent on the biomechanical properties of the eyeball, and is here set to 3 . By combining eqn (2), (4) and (5), the change in eyeball pressure as a function of the change in output voltage is:

$$
\frac{V_{\mathrm{t}}}{I_{0}} \approx A \frac{3 \rho \pi}{2 W L} \Delta p
$$

It can be seen that the output voltage displays a linear relationship with the IOP variation. Notably, micro-sized piezoresistive electrodes $(W, L)$ and large radius $(\alpha)$ could contribute to high sensitivity of the IOP sensor. The designed parameters of the as-prepared sensor are shown in Table 1.

Fig. 1c shows a schematic of the designed IOP contact lens sensor. It can be seen that two electrode coils as strain gauges are set at the lens edge and two zigzag electrode elements as fixed resistances are positioned along the radius orientation. Four electrode pads extend out for the power supply and output voltage detection. Moreover, the potential distribution of our

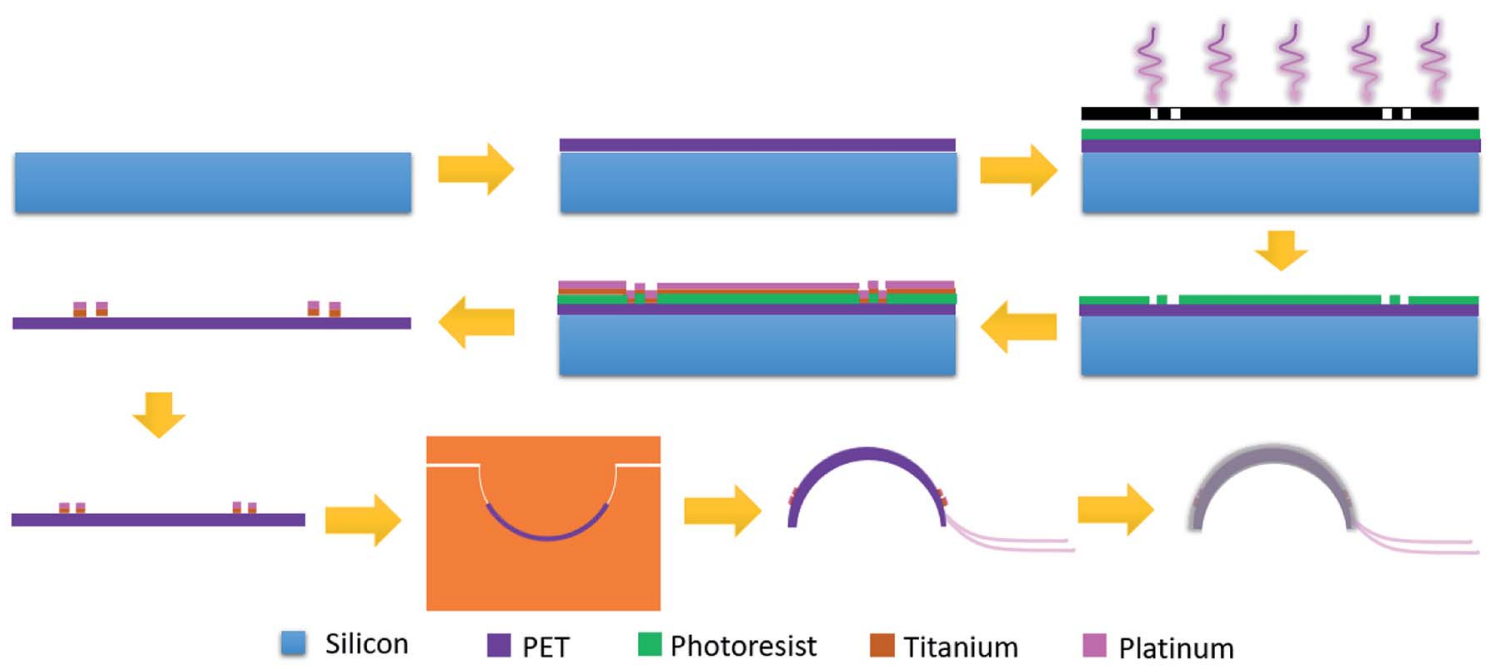

Fig. 2 Schematic diagram of the fabrication process for the IOP contact lens.
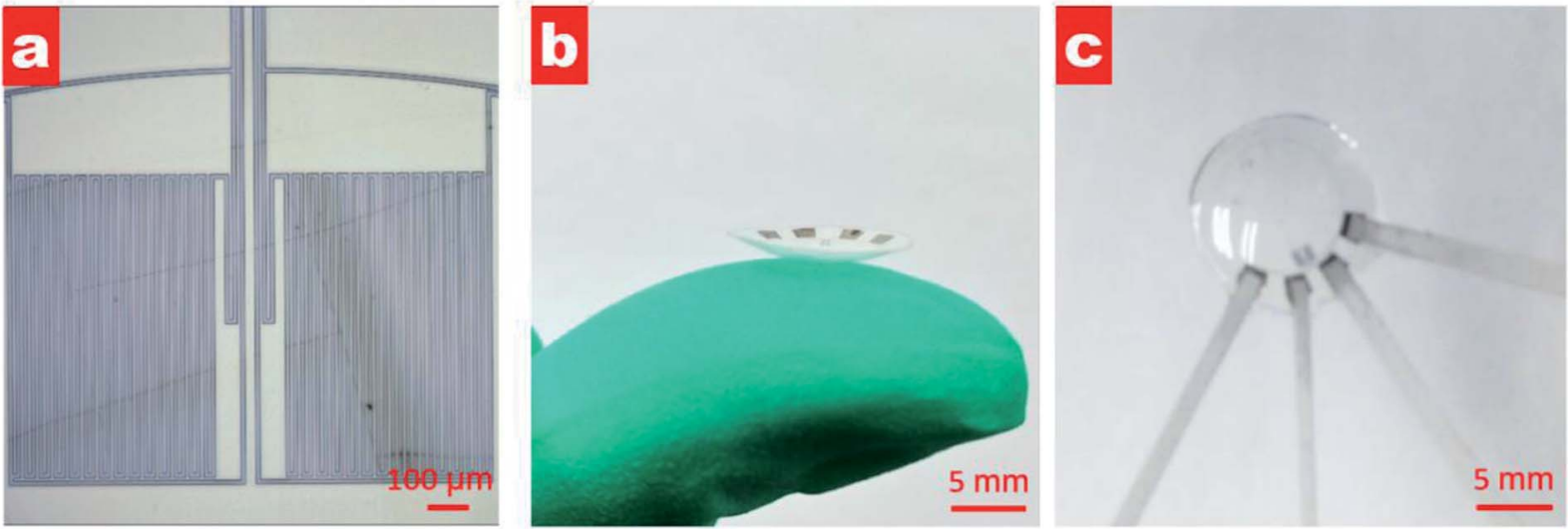

Fig. 3 (a) Microscopy image of fabricated sensor after lifting off; (b) photograph of the contact lens on a finger after thermal molding; and (c) photograph of the contact lens after PDMS packing. 


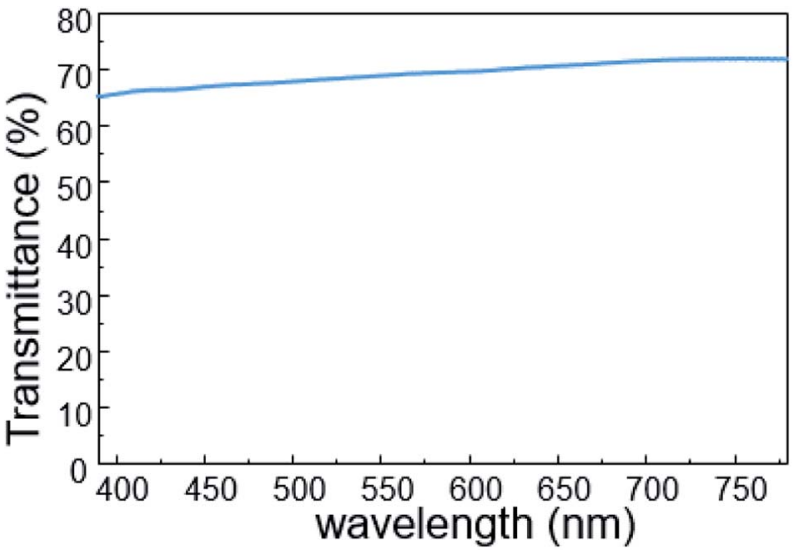

Fig. 4 The transmittance of the IOP contact lens in the visible spectrum

designed circuit has been simulated, as shown in Fig. 1d. Under a voltage supply of $5 \mathrm{~V}$ for one counterpart pair of electrode pads, the voltage in each electrode of the other counterpart pair exhibits the same value of $2.5 \mathrm{~V}$, indicating that four electrodes with the same length have zero contribution to the output voltage.

Fig. 2 shows the fabrication process of the contact lens for IOP monitoring. Firstly, a two-inch silicon wafer (Institute of Tianjin Semiconductor) was cleaned with acetone and deionized water. Flexible and transparent PET film (XFNANO, Materials Tech Co. Ltd) with a thickness of $50 \mu \mathrm{m}$ was fixed on the wafer. At a rotation speed of $3000 \mathrm{rpm}$, the positive photoresist (AZ 5350) was spin-coated onto the film and prebaked at $100{ }^{\circ} \mathrm{C}$ for $2 \mathrm{~min}$. Then the sample was exposed under a mercury lamp (H94-17 G, Sichuan Nanguang Vacuum Technology Co., Ltd) for $90 \mathrm{~s}$ and developed to form the designed pattern. After drying using the nitrogen, an oxygen plasma system (Branson IPC 3000) was used to remove the residual photoresist for $5 \mathrm{~min}$, which is beneficial to the adhesion of a deposited metal layer to the PET substrate. A titanium layer with a thickness of $10 \mathrm{~nm}$ and a platinum layer with a thickness of $50 \mathrm{~nm}$ were deposited in sequence (KJCL Lab 18, Kurt Lesker). Acetone was used to lift

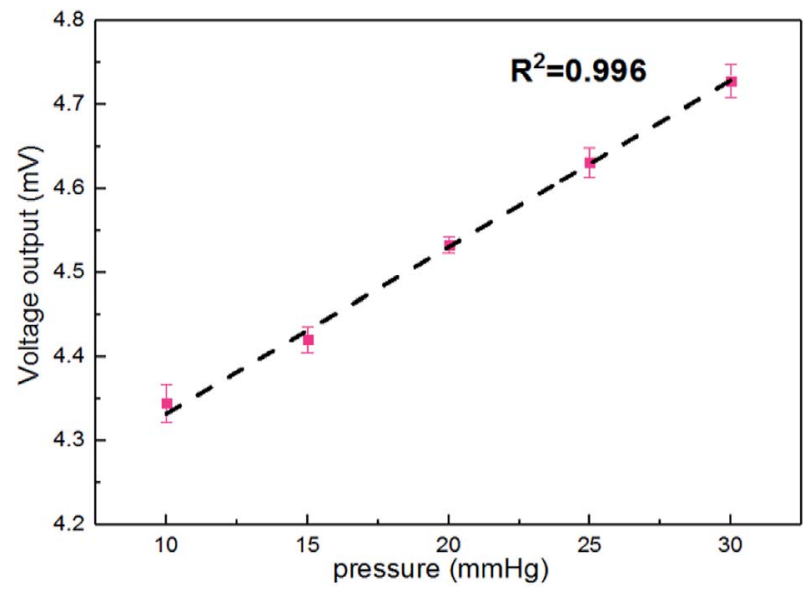

Fig. 6 The pressure in the silicone eyeball model as a function of the output voltage.

off the deposited samples and peel off the PET film from the silicon substrate.

Fig. 3a shows the microscopy image after lifting off. Two passive zigzag electrodes with width of $10 \mu \mathrm{m}$ are observed. The as-prepared resistance of each bridge is about $12.3 \mathrm{k} \Omega$. After cutting the as-prepared sensor into a circle, it was thermally molded using an aluminum model with a diameter of $10 \mathrm{~mm}$ at a temperature of $200{ }^{\circ} \mathrm{C}$, as shown in Fig. $3 \mathrm{~b}$. To get a desirable curvature, a weight of $100 \mathrm{~g}$ was put on the upper surface of the aluminum model. Aluminum foils attached by silver paste were used to lead out from the four contact pads. Finally, the sensor was encapsulated by a polydimethylsiloxane (PDMS, Sylgard 184, Dow Corning) layer using the prepolymer ingredient with base silicone and curing agent in a weight ratio of $10: 1$. PDMS has the advantages of high biocompatibility, flexibility and transparency, ${ }^{\mathbf{2 0 , 2 1}}$ and has found various applications in pressure sensors, ${ }^{22}$ microfluidics ${ }^{23}$ and artificial skin. ${ }^{24}$ As shown in Fig. 3c, the as-prepared contact lens is smooth and transparent, which would be beneficial for human optesthesia. The measured thickness of the eyeball sensor is about $120 \mu \mathrm{m}$ at the edge and about $100 \mu \mathrm{m}$ at the centre.

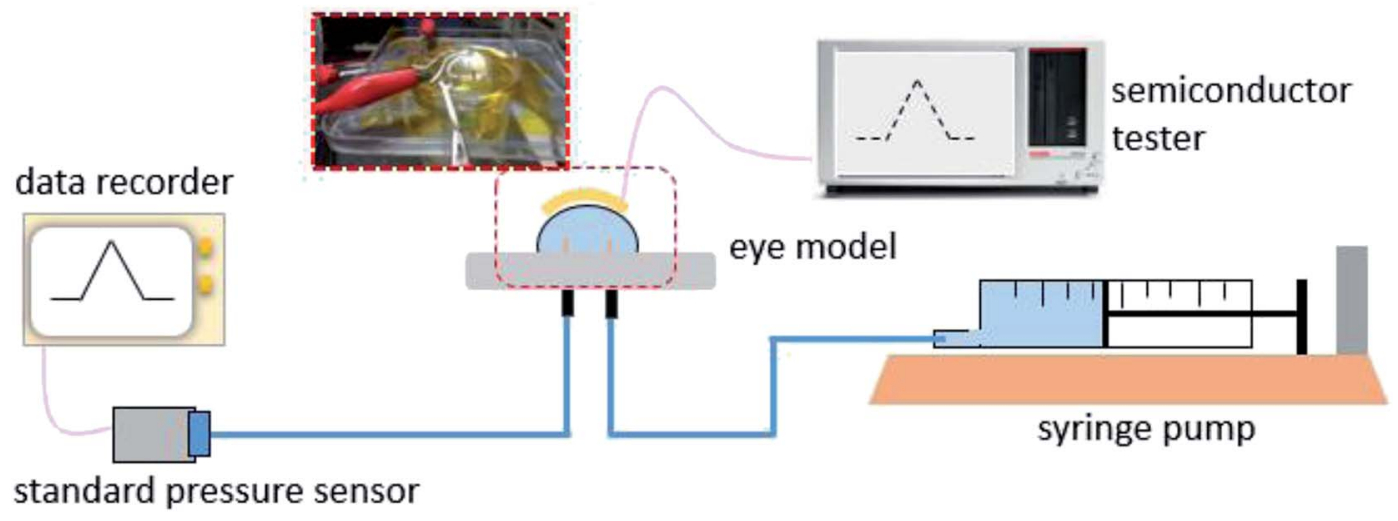

Fig. 5 The test platform for measuring performance of the contact lens sensor on the silicone eye model. 
Table 2 Comparison of our contact lens with previous reports

\begin{tabular}{|c|c|c|c|}
\hline Working principle & Invasive or non-invasive & Sensitivity & Ref. \\
\hline Microfluidic & Invasive & $137 \mu \mathrm{m} \mathrm{mmHg}{ }^{-1}$ & 9 \\
\hline Capacitive & Invasive & $15 \mathrm{kHz} \mathrm{mmHg}^{-1}$ & 10 \\
\hline Capacitive & Non-invasive & $32 \mathrm{kHz} \mathrm{mmHg}^{-1}$ & 12 \\
\hline Inductive & Non-invasive & $8 \mathrm{kHz} \mathrm{mmHg}^{-1}$ & 13 \\
\hline Piezo-resistive & Non-invasive & $1.5 \Omega \mathrm{mmHg}^{-1}$ & 14 \\
\hline Piezo-resistive & Non-invasive & $20 \mu \mathrm{V} \mathrm{mmHg}^{-1}$ & This work \\
\hline
\end{tabular}
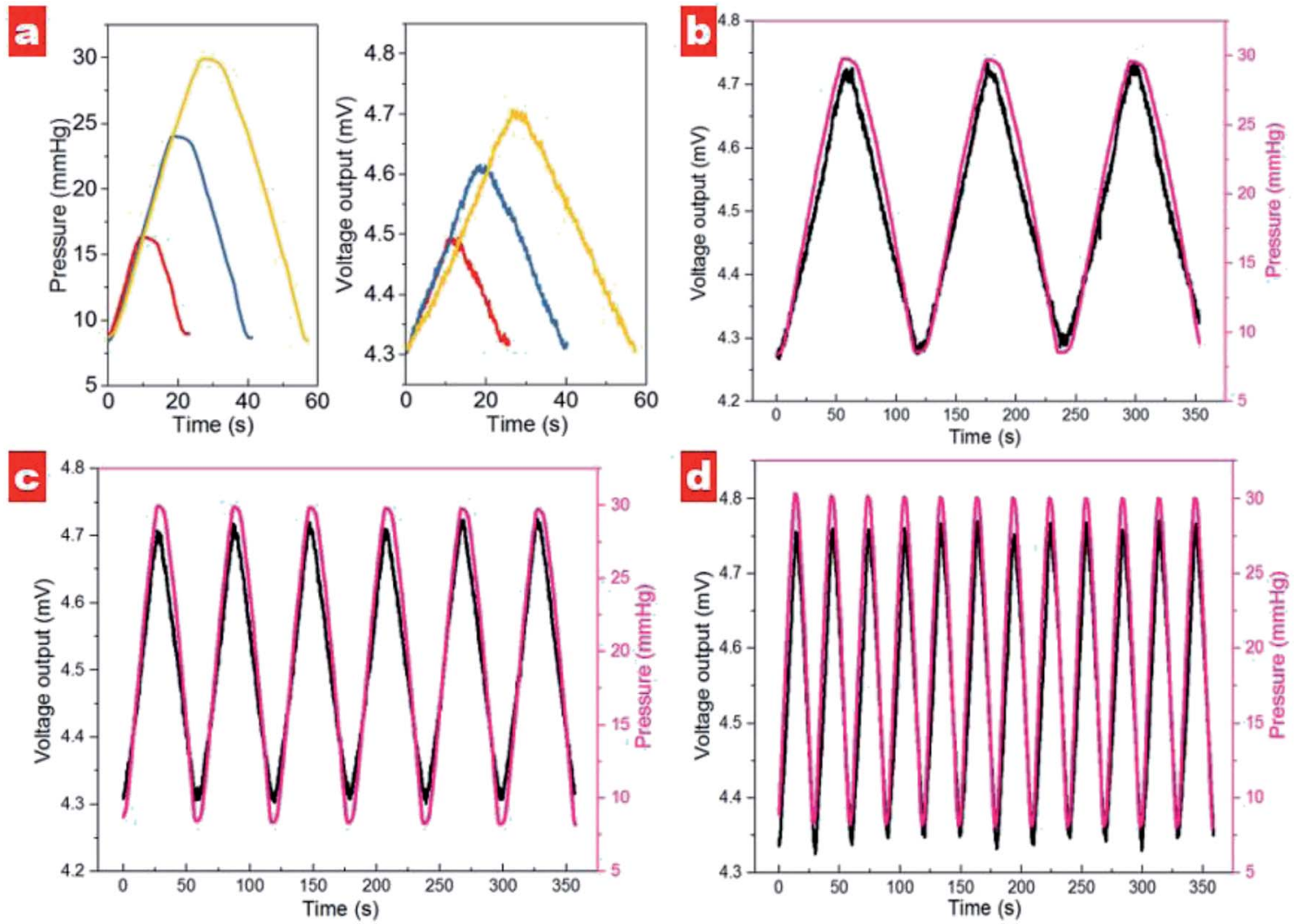

Fig. 7 The dynamic performance of the contact lens on the silicone eyeball. (a) The output voltage of the contact lens response to three different pressure ranges in the eyeball model. (b-d) The cycling performance of the contact lens at (b) low, (c) medium and (d) fast pressure variation.

\section{Results and discussion}

To investigate its visual properties, we measured the transmittance of the as-prepared contact lens in the visible spectrum, as shown in Fig. 4. The contact lens has a transmittance of about $70 \%$ in the wavelength range of $390-780 \mathrm{~nm}$, indicating adequate visual performance for potential long-term usage. Furthermore, higher transmittance can be obtained with thinner PET substrate and PDMS packing.

The real-time performance of the contact lens sensor was measured on a silicone model eye with a thickness of about 150 $\mu \mathrm{m}$ and diameter of $10 \mathrm{~mm}$, which has been widely used to investigate pressure variation in electronic eyes. ${ }^{25}$ Fig. 5 shows the home-made test system used to monitor IOP variation in the eyeball model. A syringe pump was used to inject water into the silicone eye for pressure increase and to extract water for pressure decrease. To calibrate the relationship between the output voltage and pressure variation, a standard pressure sensor (MIK-P300, Hanzhou Meacon Automation Technology, China) was used to record the pressure while water was pumped in or out. A semiconductor analyzer (Keithley 4200) was used to supply a constant current of $100 \mu \mathrm{A}$ and to detect the output voltage signal.

Fig. 6 shows the output voltages at different pressures in the silicon eyeball model. It can be seen that the output voltage is $4.34 \mathrm{mV}$ for the initial pressure of $10 \mathrm{mmHg}$ and shows 

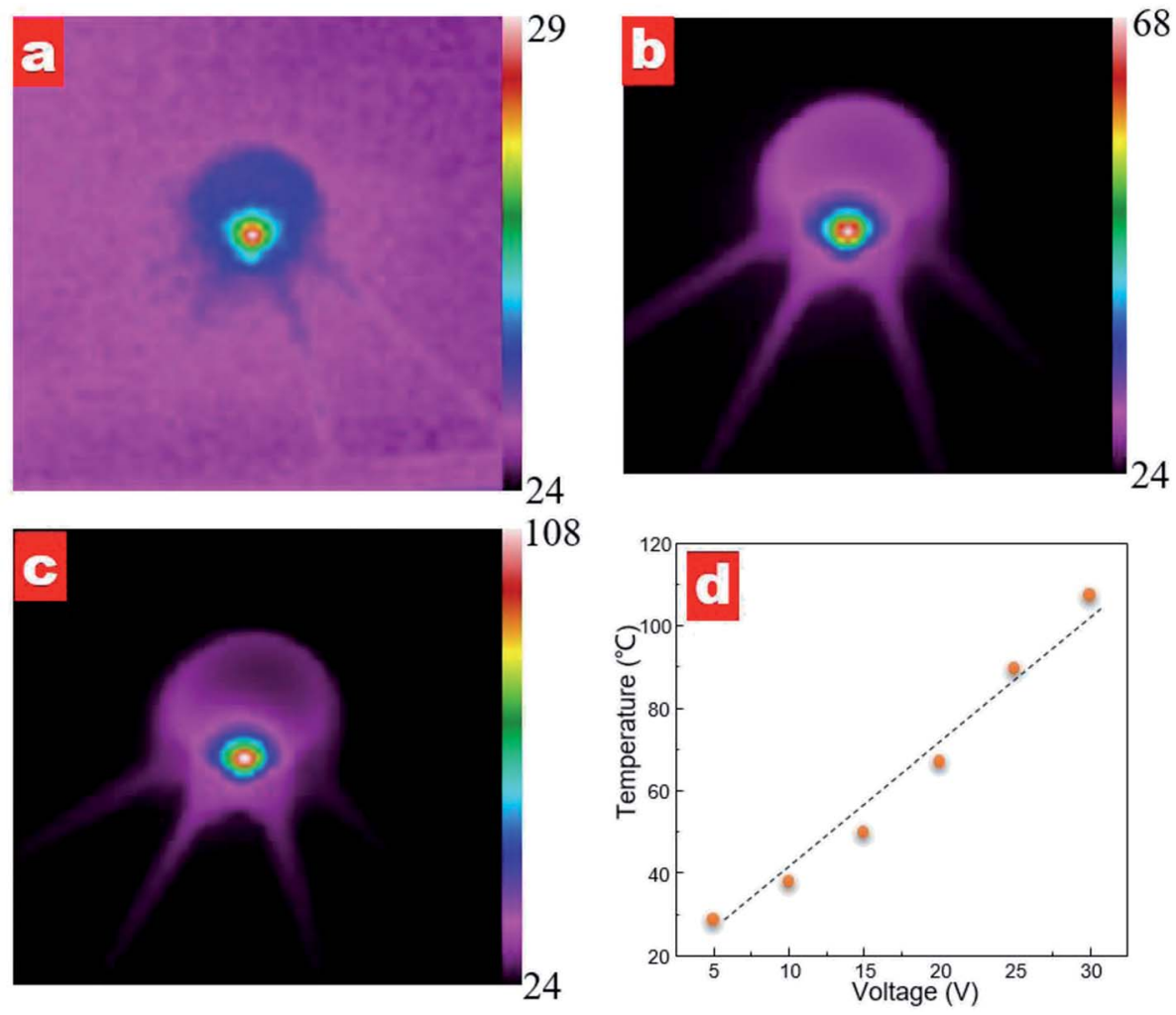

Fig. 8 The temperature distribution of the contact lens at a supplied voltage of (a) 5, (b) 20 and (c) 30 V; the color bar keys represent temperature distribution in the appropriate ranges. (d) The maximum temperature values as a function of the supplied voltage.

enhancement with increasing IOP. Moreover, the output signal exhibits an excellent linear relationship $\left(R^{2}=0.996\right)$ in the IOP range of $10-30 \mathrm{mmHg}$. The sensitivity is defined as $\left(V-V_{0}\right) /\left(V_{0} P\right)$, where $V_{0}$ and $V$ are initial output voltage and response output voltage under the changed pressure, respectively. The calculated sensitivity of the contact lens for IOP measurement is 20 $\mu \mathrm{V} \mathrm{mmHg}{ }^{-1}$. A performance comparison with previous reports is shown in Table 2.

The dynamical cycling performance of contact lens in different pressure ranges was tested, as shown in Fig. 7a. It should be noted that output voltage exhibits an excellent linear response in different pressure ranges. In the range 8.7-16.3 $\mathrm{mmHg}$, the voltage signal displays a variation of about $155 \mu \mathrm{V}$. For the measuring ranges of $8.4-24.0$ and $8.7-29.9 \mathrm{mmHg}$ the output voltages show variations of 307 and $402 \mu \mathrm{V}$, respectively. It is noted that the output voltage of the contact lens shows the same slope for pressure increase and decrease, indicating excellent recoverability. Moreover, the contact lens exhibits excellent response to different rates of pressure changes; see Fig. $7 \mathrm{~b}-\mathrm{d}$. In the range $9-30 \mathrm{mmHg}$, the contact lens shows output voltage variation that follows IOP changes well. Interestingly, with increased speed of pressure change the variation of output voltage shows the decreased values of 440,414, and $408 \mu \mathrm{V}$ for slow, medium and fast speeds, respectively. We suppose that high speed of injection and extraction would reduce the effective response time for each cycle and contribute to the small fluctuation of output signals.
To investigate the heating effect of different power supplies, we measured the temperature distribution of the contact lens, as shown in Fig. 8. When a voltage supplied to the contact lens, the sensor shows an instant heating response. Fig. 8a-c shows the temperature distribution images at a supplied voltage of 10,20 and $30 \mathrm{~V}$, respectively. It can be seen that a highlight heating area is observed in the zigzag resistance region for all supplied voltages while no obvious heating area is observed along the active strain gauges. The measured maximum temperature values are $37.8,67.1$ and $107.3{ }^{\circ} \mathrm{C}$ with voltage supplies of 10, 20 and $30 \mathrm{~V}$, respectively. Furthermore, the maximum temperature varies linearly as a function of the supplied voltage; see Fig. 8d. The calculated ratio of temperature versus voltage is $3.0^{\circ} \mathrm{C} \mathrm{V}^{-1}$.

\section{Conclusions}

In conclusion, we have developed a non-invasive contact lens for IOP monitoring based on the transparent substrate PET. A Wheatstone bridge was designed to improve the detection of weak deformation of the eyeball, which is beneficial for high sensitivity and precision. Via lithography and deposition processes, two active strain gauges and two passive strain gauges were fabricated. A thermal model was used to form a contact lens shape, which was then encapsulated in a biocompatible PDMS layer. In the visible spectrum the contact lens exhibits a transmittance of $70 \%$. The calculated sensitivity 
of the contact lens is $20 \mu \mathrm{VmmHg}{ }^{-1}$ in the IOP range of $10-30$ $\mathrm{mmHg}$. Moreover, it exhibits excellent dynamic response to different speeds of IOP pressure variation. The contact lens shows promising application in continuous IOP monitoring, which would be beneficial in the diagnosis and therapy of glaucoma patients.

\section{Conflicts of interest}

There are no conflicts to declare.

\section{Acknowledgements}

This work was supported by the National Key R\&D Program (2016YFA0200400), National Natural Science Foundation (61434001, 61574083, 61874065, 51861145202), and National Basic Research Program (2015CB352101) of China. The authors are also thankful for the support of the Research Fund from the Beijing Innovation Center for Future Chip (KYJJ2018005), Beijing Natural Science Foundation (4184091), and Shenzhen Science and Technology Program (JCYJ20150831192224146).

\section{Notes and references}

1 H. A. Quigley and A. T. Broman, Br. J. Ophthalmol., 2006, 90, 262-267.

2 D. Pascolini and S. P. Mariotti, Br. J. Ophthalmol., 2010, 96, 614-618.

3 H. K. J. Liu, K. Mansouri and R. N. Weinreb, PLoS One, 2015, 10, e0129529.

4 K. Mansouri, R. N. Weinreb and F. A. Medeiros, Semin. Ophthalmol., 2013, 28, 157-164.

5 J. Choi, K. H. Kim, J. Jeong, H. S. Cho and M. S. Lee, Invest. Ophthalmol. Visual Sci., 2007, 48, 104-111.

6 B. E. Klein, R. Klein and K. L. Linton, Invest. Ophthalmol. Visual Sci., 1992, 33, 2224-2228.

7 N. A. Loewen, J. H. K. Liu and R. N. Weinreb, Invest. Ophthalmol. Visual Sci., 2010, 51, 933-937.

8 E. Hughes, P. Spry and J. Diamond, J. Glaucoma, 2003, 12, 232-236.
9 I. E. Araci, B. Su, S. R. Quake and Y. Mandel, Nat. Med., 2014, 20, 1074-1078.

10 G. Chitnis, T. Maleki, B. Samuels, L. B. Cantor and B. Ziaie, IEEE Trans. Biomed. Eng., 2013, 60, 250-256.

11 P. J. Chen, S. Saati, R. Varma, M. S. Humayun and Y. C. Tai, MEMS IEEE 22nd International Conference on Micro Electro Mechanical Systems, 2009, pp. 244-247.

12 G. Z. Chen, I. S. Chan and D. C. C. Lam, Sens. Actuators, A, 2013, 203, 112-118.

13 G. Z. Chen, I. S. Chan and D. C. C. Lam, Med. Eng. Phys., 2014, 36, 1134-1139.

14 V. Laukhin, I. Sánchez, A. Moya, E. Laukhina, R. Martin, F. Ussa, C. Rovira, A. Guimera, R. Villa and J. Aguiló, Sens. Actuators, A, 2011, 170, 36-43.

15 M. Leonardi, P. Leuenberger, D. Bertrand, A. Bertsch and Ph. Renaud, TRANSDUCERS, 12th International Conference on Solid-State Sensors, Actuators and Microsystems, 2003, vol. 2, pp. 1043-1046.

16 M. Leonardi, P. Leuenberger, D. Bertrand, A. Bertsch and Ph. Renaud, Invest. Ophthalmol. Visual Sci., 2004, 45, 31133117.

17 M. D. Twa, C. J. Roberts, H. J. Karol, A. M. Mahmoud, P. A. Weber and R. H. Small, J. Glaucoma, 2010, 19, 382-390.

18 A. K. C. Lam and W. A. Douthwaite, Ophthal. Phys. Opt., 1997, 17, 18-24.

19 A. Thanachayanont and S. Sangtong, ETRIJ., 2007, 29, 70-78. 20 X. Liu, Y. Zhu, M. Nomani, X. Wen, T. Y. Hsia and G. Koley, J. Micromech. Microeng., 2013, 23, 025022.

21 D. Baker, X. Liu, H. Wang, C. Luo and L. Tang, Biomacromolecules, 2011, 12, 997-1005.

22 Y. Pang, K. Zhang, Z. Yang, S. Jiang, Z. Ju, Y. Li, X. Wang, D. Wang, M. Jian, Y. Zhang, R. Liang, H. Tian, Y. Yang and T. L. Ren, ACS Nano, 2018, 12, 2346-2354.

23 A. R. Abate, D. Lee, T. Do, C. Holtze and D. A. Weitz, Lab Chip, 2008, 8, 516-518.

24 H. K. Lee, S. I. Chang and E. Yoon, J. Microelectromech. Syst., 2006, 15, 1681-1686.

25 I. Jung, J. Xiao, V. Malyarchuk, C. Lu, M. Li, Z. Liu, J. Yoon, Y. Huang and J. A. Rogers, Proc. Natl. Acad. Sci. U. S. A., 2011, 108, 1789-1793. 